\title{
Development of a Precise Broken End Positioning and Indicating System
}

\author{
Shuai Xu ${ }^{1, a}$, Xiaofang Yang ${ }^{1, b}$, Ting Zhu ${ }^{1, c}$, Guixiang Yao ${ }^{1, d}$, \\ and Chunmo Wang ${ }^{1, e}$
}

\author{
${ }^{1}$ No. 285 South Jiefang Rd. Yancheng Institute of Industry Technology, \\ Yancheng(224005), Jiangsu, China

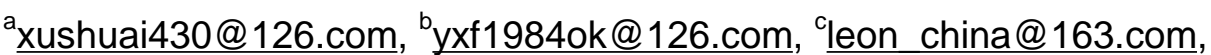 \\ yaoguixiang8067199@126.com, ${ }^{\mathrm{e}}$ wangchunmo@126.com
}

Keywords: warp break detector, precise positioning, dropper bar, resistance measurement Abstract: Modern looms can usually detect a broken warp yarn by using a warp break detector, but they cannot locate the broken end precisely and automatically. High-count and high-density fabrics with a broad width usually have a warp consisting of up to ten thousand of ends that are closely packed. This makes it very difficult for a worker to locate the broken end quickly. In order to solve this problem, the authors developed a precise broken end positioning and indicating system. It has advantages such as high operational stability, high positioning accuracy, and clear visual indication of failures.

\section{Foreword}

Most modern looms have a warp stop motion to stop the machine immediately when a warp yarn is broken. Currently, a warp break detector of the warp stop motion can detect any broken warp yarn by using the droppers, but it cannot visually show its location and a worker has to locate it manually. For the high-count and high-density fabric with a broad width, as there can be up to ten thousand warp ends, it is even more difficult to find the position of the broken end. In order to solve this problem, the authors developed a new system to precisely locate the broken ends.

\section{The Principle of Positioning}

\section{Basic Principles of Warp Break Detector}

The most commonly used contact warp break detector consists of a dropper bar and droppers. The structure of a typical dropper bar is shown in Fig. 1. It is composed of an electrode 1, an electrode 2 and an insulating layer 3. The insulating layer 3 is between the electrode 1 and the electrode 2 and it separates them, so the electrode 1 and electrode 2 are insulative to each other.

Fig. 2 shows the operating principle of the contact warp break detector. When a warp end 5 is broken or becomes too loose, the dropper falls and electrode 1 and electrode 2 become conductive to each other. At this time, a signal is sent out and the loom stops immediately. Electrode 1 is mostly made of brass, and the electrode 2 and dropper 4 are made of stainless steel.

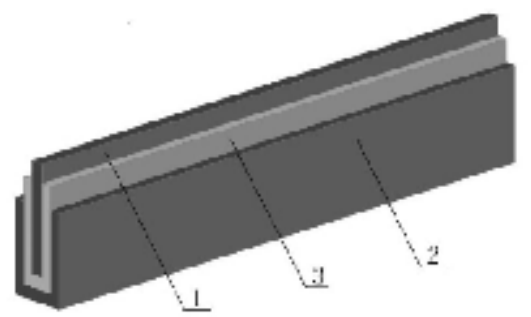

Fig. 1. Structure of dropper bar

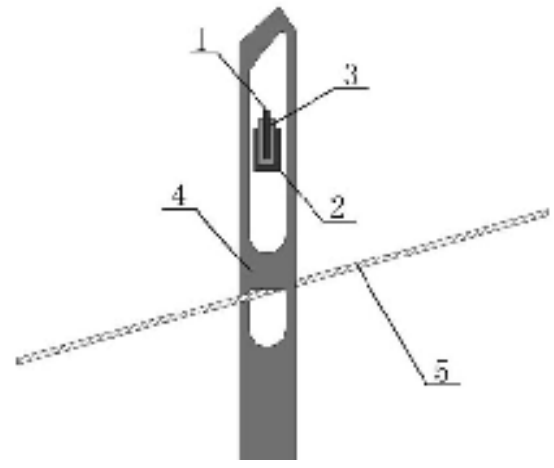

Fig. 2. Operating principle of contact warp break detector 


\section{Principle of Resistance Positioning}

The model of a contact warp break detector can be simplified as shown in Fig. 3(a). When the electrode 1 and electrode 2 become conductive by the dropper 4, the distance between dropper 4 and the loom's right end and the left end are marked as $\mathrm{X}$ and $\mathrm{Y}$ respectively. Then, the corresponding electric circuit diagram of Fig. 3(a) is shown in Fig. 3(b). In Fig. 3(b), $R_{\mathrm{C} 1}$ and $\mathrm{R}_{\mathrm{C} 2}$ represent the contact resistances between the dropper 4 and the electrode 1, and the dropper 4 and the electrode 2 respectively. $R_{W}$ is the resistance of the dropper 4 itself. $R_{1 X}$ is the resistance produced by the part $X$ of the electrode 1 , and $R_{1 Y}$ is produced by part $Y$. Similarly, $R_{2 X}$ and $R_{2 Y}$ are created in the same way. If any of $R_{1 X}, R_{1 Y}, R_{2 X}$ and $R_{2 Y}$ 's values is obtained, the distances $X$ and $Y$ can be calculated by the law of resistance, e.g. the value of $R_{2 X}$ is obtained, the distance $X$ can be calculated and determined as follows.

Let electrode 2's resistivity be $\rho$, cross section area be $S$, then there is:

$$
R_{2 X}=\frac{\rho X}{S}=k X
$$

In the formula, $k$ can be treated as unit length resistance of a material and it can be measured. Its unit is $\Omega / \mathrm{m}$. So, the value of $\mathrm{X}$ would be:

$$
L=\frac{R_{2 X}}{k}
$$

If the formula is further analyzed, we come to a conclusion that in order to make the $\mathrm{X}$ accurate, $k$ and $\mathrm{R}_{2 \mathrm{X}}$ have to be accurate in the first place.

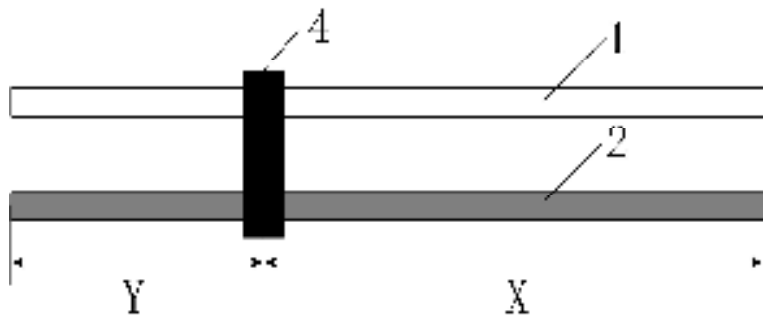

(a) model

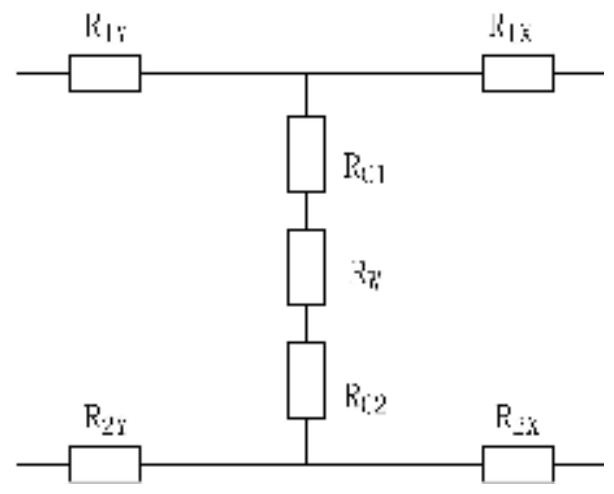

(b) circuit diagram

Fig. 3. Model and circuit diagram of warp break detector

\section{Design of a New End Break Detector}

To design a precise broken end positioning and indicating system, the structure of the warp break detector must be redesigned to ensure the values of $\mathrm{k}$ and $\mathrm{R}_{2 \mathrm{X}}$ are as reliable as possible. And after that, a matching resistance measuring system can be designed. The value of $\mathrm{k}$ needs to be constant and cannot be too small. The reason of that is explained as follows.

Suppose in one measurement, the $\mathrm{R}_{2 \mathrm{X}}$ in Fig. 3 is measured $R i$, and the error margin is $\Delta R$. The actual value is $R_{0}$, which equals the resistance value of length $X_{0}$ of electrode 2 . Then we come to the following formula (3).

$$
R_{i}=R_{0}+\Delta R
$$

Based on formulas (2) and (3), let the measured length be $X i$, the value of $X i$ will be:

$$
\mathrm{X}_{i}=\frac{R_{i}}{k}=\frac{R_{0}+\Delta R}{k}=X_{0}+\frac{\Delta R}{k}
$$

If $X i$ is used as the final result to determine the position of the broken end, the error will be: 


$$
\Delta X=X_{i}-X_{0}=\frac{\Delta R}{k}
$$

It is generally known that the error of a measurement is random and has a constant range, and it will not dwindle down as the measured values get smaller. From formula (5), the smaller $k$ is, the greater the positioning error will be. Besides, it is more difficult to get an accurate measurement for smaller resistances. For these reasons the choice of electrode depends upon the material's $k$ value, i.e. the unit length resistance. And a greater $k$ value will be better to the system.

As mentioned above, currently, the materials of the dropper bar are brass and stainless steel, and the $k$ is very small. This leads to the fact that the resistance is only $1-2 \Omega$ for the full length of the electrode, which is usually 2-4 meters long. This will be a disadvantage for measuring the resistance. Therefore, changes must be made to the materials and structure of the electrode. Fig. 4 shows the structure of the newly designed dropper bar. The material for electrode 1 remains unchanged, brass, However, the electrode 2, which is attached to the insulating layer 3, no longer uses stainless steel. Instead, a $0.1 \mathrm{~mm}$ diameter $\mathrm{Cr} 20 \mathrm{Ni} 80$ nichrome wire is used. The wire's resistance per unit length is $138.8 \Omega / \mathrm{m}$. It also has good physical properties such as good resistance to abrasion and corrosion, which make it ideal for the new positioning system.

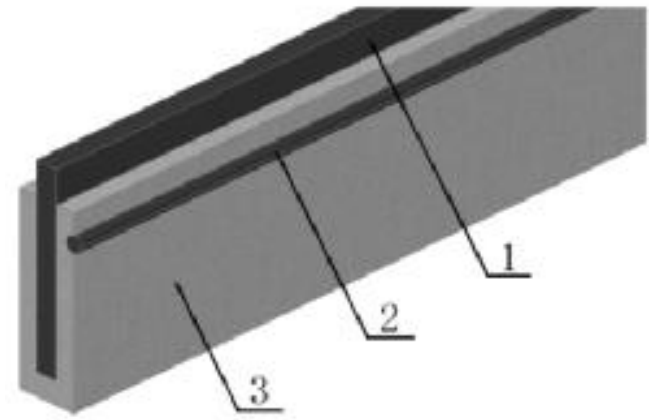

Fig. 4. Structure of new dropper bar

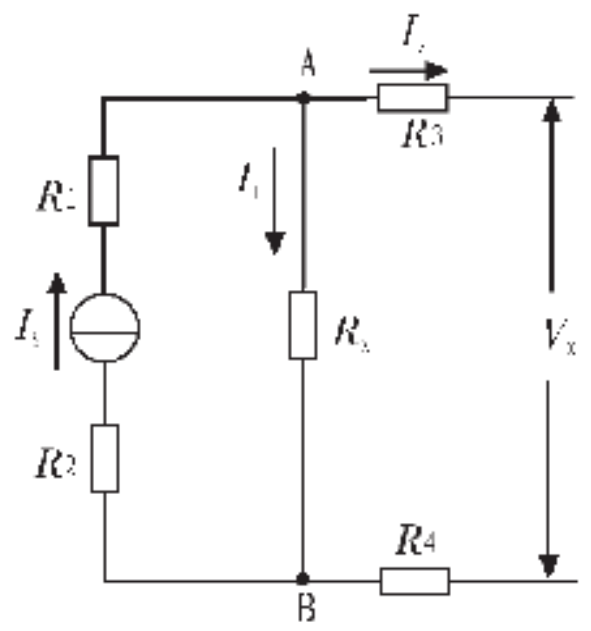

Fig. 5. Four-wire method

\section{Design of the System}

\section{Methods of Resistance Measurement}

The model and the circuit diagram shown in Fig. 3 can still be used for the new dropper bar shown in Fig. 4. Now obtaining the accurate resistance value for $\mathrm{R}_{2 \mathrm{X}}$ is crucial to precise positioning. Because the dropper bar is full of droppers when in operation, it would be difficult to directly connect two wires to both ends of $\mathrm{R}_{2 \mathrm{X}}$. If it is directly measured from the right end, errors will occur because resistance values of $R_{C 1}, R_{C 2}, R_{W}$ and $R_{1 X}$ are also included. In order to solve this problem, this paper adopts the four-wire method, which is generally accepted as the best way to measure resistance. The principle of the four-wire method is shown in Fig. 5.

In Fig. 5, Is is a constant current source. Rx is the resistance to be measured. A and B are the two voltage measuring points. $\mathrm{R}_{1}, \mathrm{R}_{2}, \mathrm{R}_{3}$ and $\mathrm{R}_{4}$ are the resistances of the four wires. From Fig. 5 , we have formula (6).

$$
I_{S}=I_{1}+I_{2}
$$

When the signal is amplified by an instrumentation amplifier, the input impedance of the amplifier is extremely high and its bias current is extremely small, that means in formula (6): $I_{2} \approx 0, I_{S} \approx I_{1}$.

Because $I_{2} \approx 0$, the decrease of voltage caused by the wires $R_{3}$ and $R_{4}$ is negligible. So $V_{X}$ equals the voltage on $R_{X}$. From this, the accurate value of $R_{X}$ can be calculated by $R_{X}=V_{X} / I s$. 
Similarly, the new method for resistance measurement based on the four-wire method is shown in Fig. 6. Although the resistances of $\mathrm{R}_{\mathrm{C} 1}, \mathrm{R}_{\mathrm{C} 2}, \mathrm{R}_{\mathrm{W}}$ and $\mathrm{R}_{1 \mathrm{X}}$ are unknown, the incurring voltage decrease of them can be neglected for the same reason discussed above. Finally, we come to the conclusion that the input voltage on the instrumentation amplifier is exactly equal to the voltage of $R_{2 X}$.

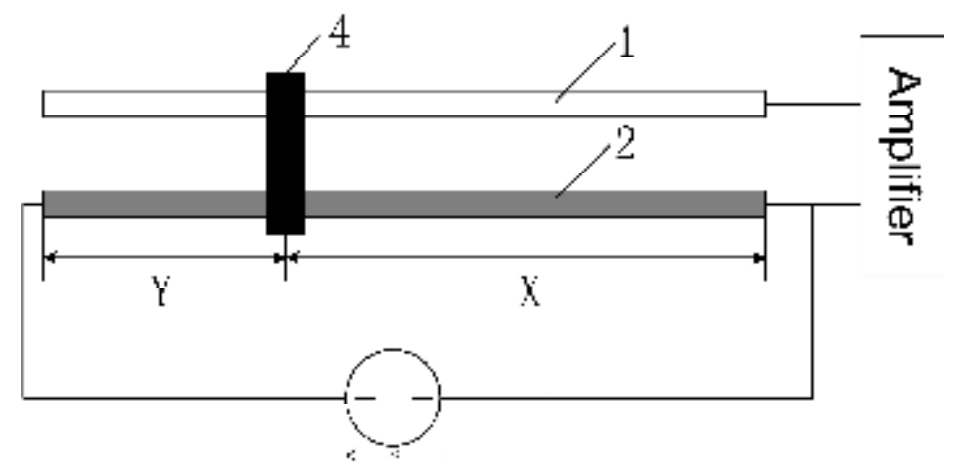

Fig. 6. Voltage measurement of $\mathrm{R}_{2 \mathrm{X}}$

\section{Overall Design of the System}

The overall structure of the system is shown in Fig. 7. Below is how it works. First, the system uses the original circuit and photocoupler of the loom, which are used to check if there is a broken end. When a broken end is detected, the power of the checking circuit is cut by the relay. Meanwhile, the relay provides power to the constant current source and the signal processing circuit, which makes it possible to measure the resistance. After that, the resistance is converted to a distance between the broken end and the right end of the loom by the MCU, and finally the position of the broken end is determined based on the distance and is shown by an indicator.

The function of the relay is to switch between two directions of power supply, one is to the original checking circuit and the other is to the constant current source circuit and the signal processing circuit. The circuits of the two directions are separated and do not interfere with each other. Besides, as shown in Fig. 7, the checking circuit's wire is connected to the left end of the bar, and the signal processing circuit's wire is connected to the right end.

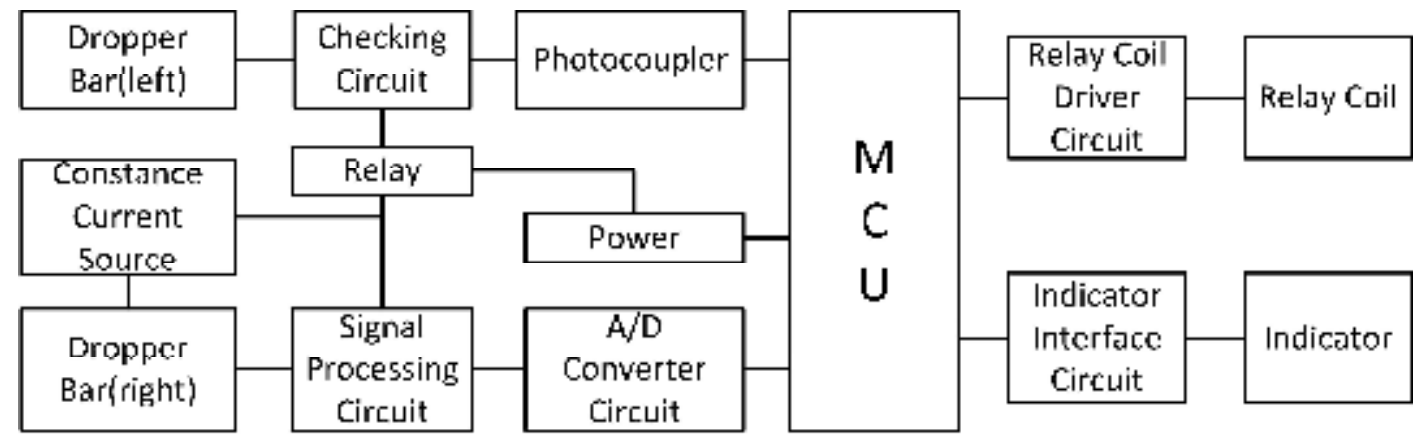

Fig. 7. System structure

\section{Design of System Hardware}

\section{Design of Constant Current Source Circuit}

The constant current source of the system mainly consists of an ultralow offset voltage operational amplifier OP07 and a high precision resistor. The amplifier's control voltage is from the REF pin of ADC MAX197. The constant current circuit and A/D converter circuit use the same reference voltage, which means the drift of reference voltage does not affect the measurements, and the designing of the constant current source can be simplified. In the constant current circuit, shown in Fig. 8, the current is calculated as $\mathrm{I}=4.096 \mathrm{~V} / 2 \mathrm{k} \Omega=2.048 \mathrm{~mA}$. As the resistor's temperature coefficient and precision will significantly affect the circuit, a high precision resistor is recommended here. 


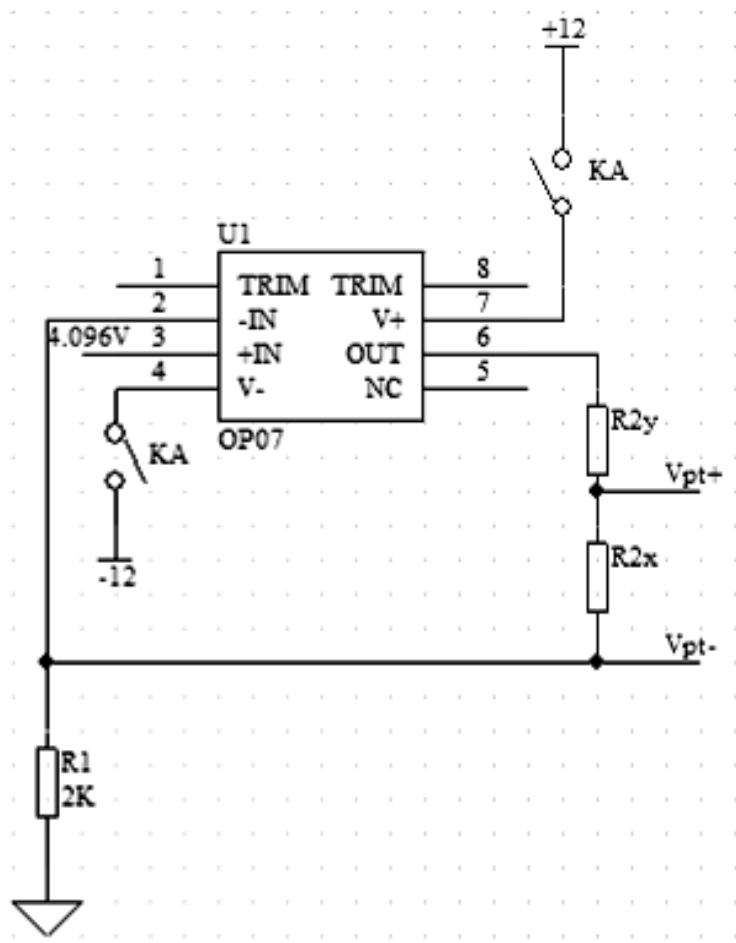

Fig. 8. Constant current source circuit

\section{Design of Signal Processing Circuit}

As shown in Fig. 9, the signal processing circuit in this design involves two operational amplifiers, AD620 and OP07. AD620 is an instrumentation amplifier with high precision and stability. Its input impedance is up to $10 \mathrm{G} \Omega$, and the bias current is as low as $2 \mathrm{nA}$. The gain setting of AD620 is as simple as adding an external resistor $R_{G}$ between pin 1 and pin 8 . The gain equation is $G=1+$ $49.4 \mathrm{k} \Omega / \mathrm{R}_{\mathrm{G}}$. The length of the dropper bar varies on different types of looms, but is usually less than four meters, which makes the value of $\mathrm{R}_{2 \mathrm{X}}$ in Fig. 3 fall in $0 \Omega \sim 555.2 \Omega$. And in the constant current source circuit, the voltage of $R_{2 X}$, i.e. the input voltage of signal processing circuit, is $0 \mathrm{mV} \sim 1137 \mathrm{mV}$. However, the output voltage of signal processing circuit must be $0 \mathrm{~V} \sim 5 \mathrm{~V}$. For this reason, the voltage needs to be converted by a proper gain $\mathrm{G}$. That is, $\mathrm{G}=5 / 1.137 \approx 4.4$.

\section{Design of A/D Converter Circuit}

The A/D converter used in the design is a 8-channel, 12-bit converter MAX 197. The model of the MCU is AT89S52. The connection of them is shown in Fig. 10. MAX 197 runs in internal clock mode in this design and uses the internal reference. REF pin outputs a reference voltage of $4.096 \mathrm{v}$ that is used as the control voltage by the constant current source.

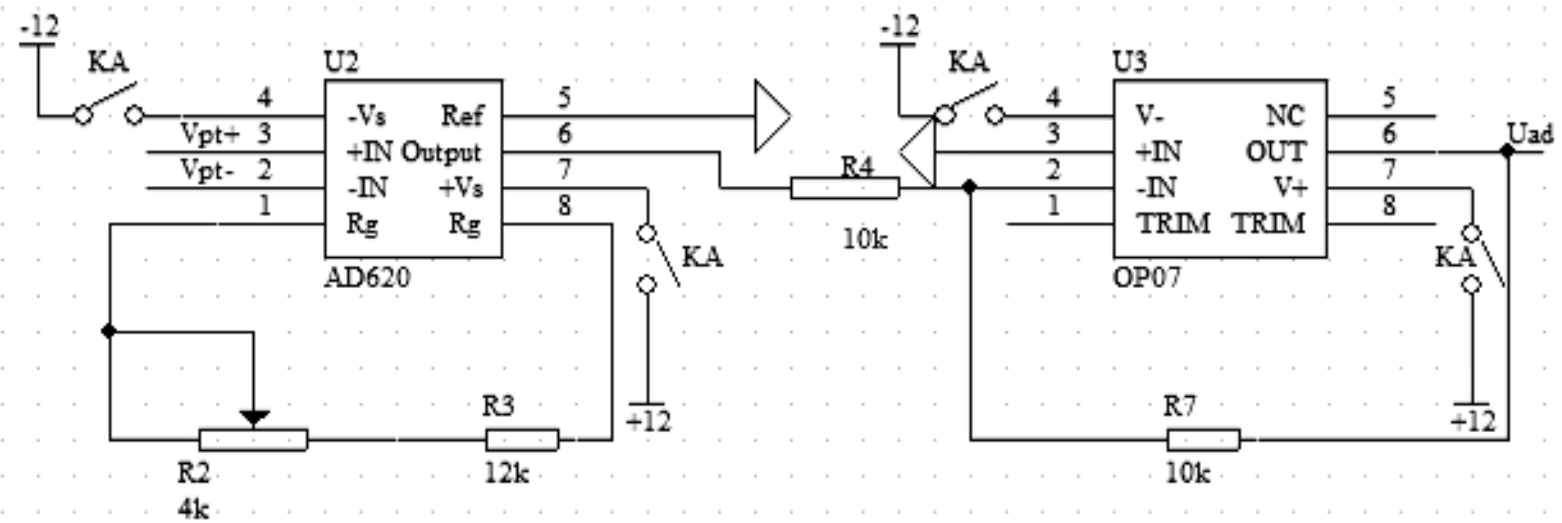

Fig. 9. Signal processing circuit 


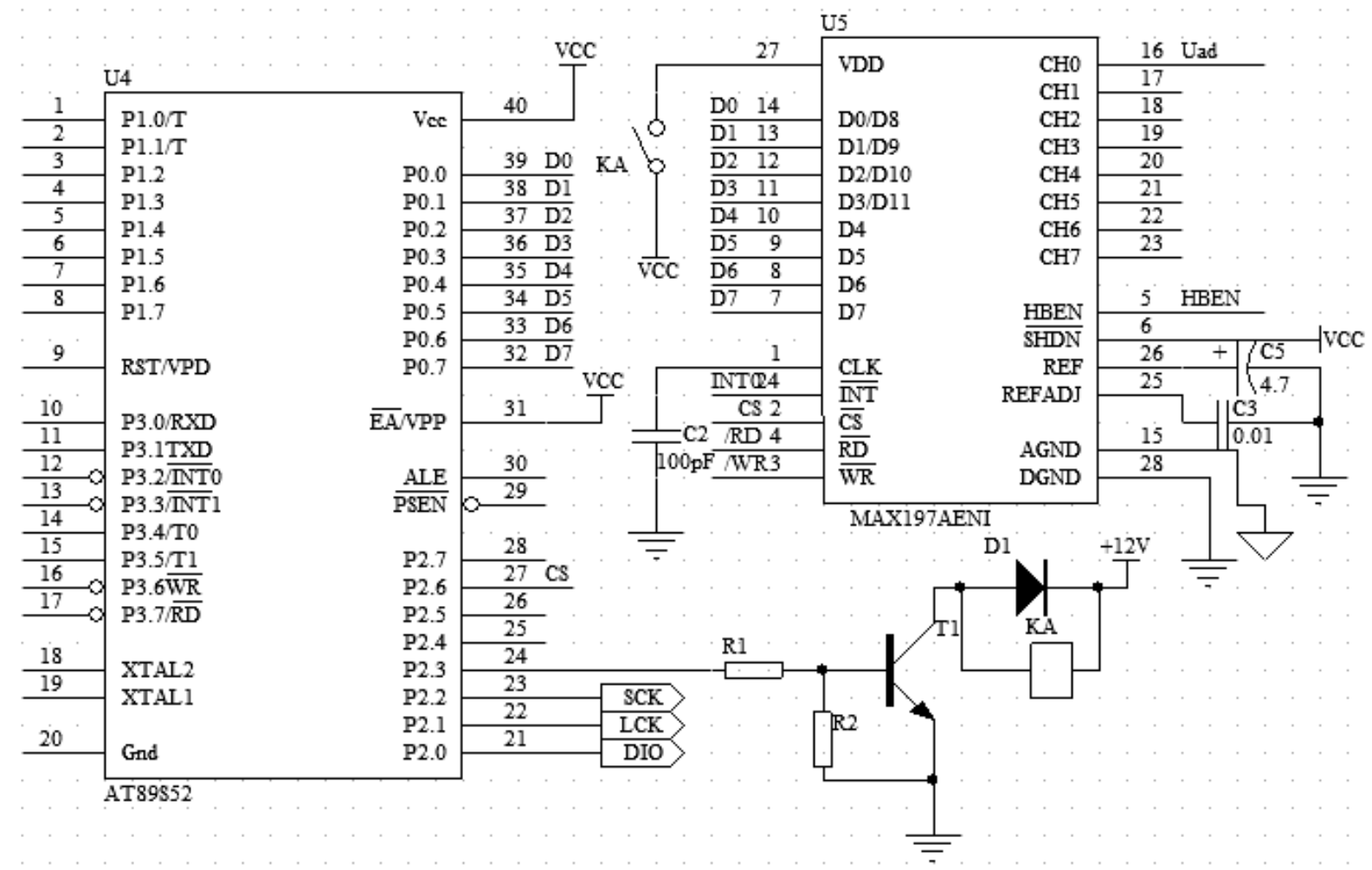

Fig. 10. A/D converter circuit

\section{Design of Indicator Circuit}

The indicator of the system is a long PCB with a row of LED bulbs on it, and its length equals the length of the dropper bar. All the LEDs on the PCB are spaced $1 \mathrm{~cm}$ apart to indicate the position of the broken end. Considering the length of the dropper bar on most looms, the number of LEDs of the indicator ranges from 200 to 400. In order to control all these LEDs, two 74HC595 registers are used to create an extendable indicator, of which the length is adjustable. The connection of 74HC595 registers and the LEDs is shown in Fig. 11. In the figure, two 74HC595 registers control 64 LEDs. Both the registers and the LEDs are installed on a $64 \mathrm{~cm}$ long PCB, which can be extended according to the length of the dropper bar.

\section{Design of System Software}

The difficulty of system software designing is how to timely switch between the checking program which is used to determine if there is a broken end and the positioning program which is used to locate it. The flow chart of the program is shown in Fig. 12. When the program runs, a flag bit is created to control the relay. It is also used to prevent the relay from operating again after a broken end has been located and displayed. The flag bit and the indicator are initialized by an interrupt program when the start button of the loom is pressed. Besides, the A/D conversion process in the program is supposed to be executed multiple times in order to get better results.

In the distance calculation, as mentioned earlier, the maximum length of the dropper bar is four meters, and the PCB has the same length with 400 LEDs on it. Suppose the result of A/D conversion is $V_{0}$ in a measurement, then the $X$ in the Fig. 3 is calculated by the formula $X=V_{0} \times 400 / 4096$, and the unit of $\mathrm{X}$ is $\mathrm{cm}$. As $\mathrm{X}$ represents the distance between the broken end and the right end of the dropper bar, the LED for the broken end is determined and lights up. 

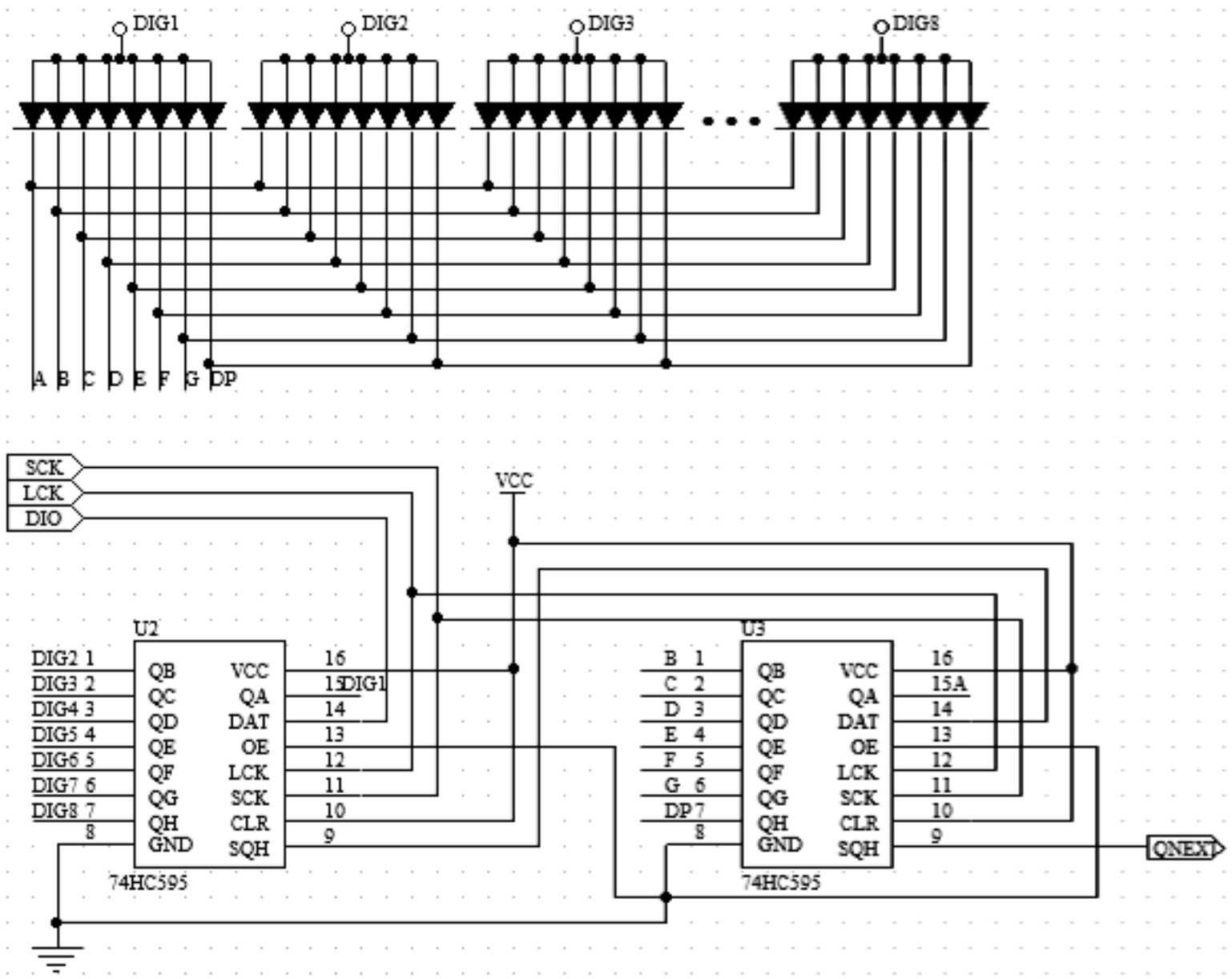

Fig. 11. Indicator circuit

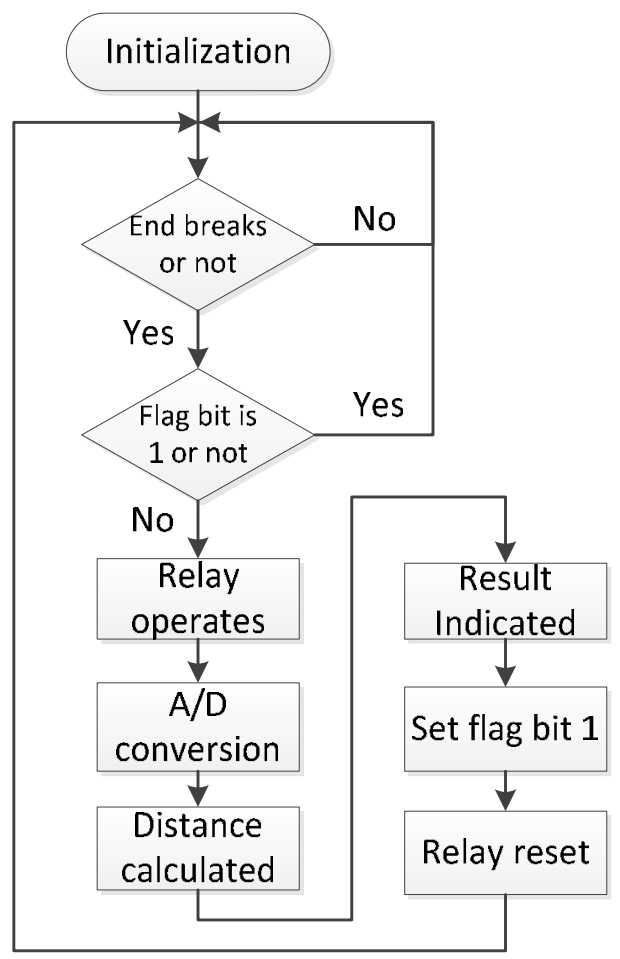

Fig. 12. Flow chart of the program 


\section{Conclusions}

The system works on the basis of law of resistance, uses a nichrome wire as the electrode and adopts the four-wire method for resistance measurement. The system includes a constant current source based on the ADC reference, a high-precision instrumentation amplifier, and an extendable LED indicator. The system runs stably and indicates visually. Practical applications have shown that the system is able to precisely locate the broken end, and therefore it significantly improves the operation efficiency of the workers and reduces damage to other warp ends.

\section{Acknowledgements}

This work was financially supported by Industry-university-research Cooperation Prospective Project of Jiangsu (BY2014112) and Top-notch Academic Programs Project of Jiangsu Higher Education (PPZY2015C254).

\section{References}

[1] Zhang Xiutai, Hu Xuehui, Zhai Yafang: Chinese Journal of Sensors and Actuators. Vol. 23 No.6 (June 2010), p. 812-815.

[2] LIU Yun, LIN Xue-yan: Electromechanical Components, 6(2) (2008), p. 23-26.

[3] SUNAMID, MCNAMESJ, COLBERTA, etal. Variable frequency bioimpedance instrumentation[C]. Proceeding 26th Ann. Inter. Conf. IEEE Eng. Med. Biol. Soc., 2004(4):2386-2389..

[4] ROSSA S. Current source design for electrical impedance tomography[J]. Physiological Measurement, 2003, 24(2):509-516.

[5] LEEJW. Precision constant current source for electrical impedance tomography[J]. Proceeding of 25th Annual International Conference of the IEEE EMBS, Cancun, Mexico, 2003:17-21.

[6] Zhao Shaobo: Chinese Journal of Scientific Instrument, Vol. 22 No.4 (August 2001), p. 136-138 